\title{
Functional analysis of four LDLR 5'UTR and promoter variants in patients with familial hypercholesterolaemia
}

\author{
Amna Khamis ${ }^{1}$, Jutta Palmen ${ }^{1}$, Nick Lench ${ }^{2}$, Alison Taylor ${ }^{2}$, Ebele Badmus ${ }^{1}$, Sarah Leigh ${ }^{1}$ \\ and Steve E Humphries ${ }^{\star 1}$
}

Familial hypercholesterolaemia (FH) is an autosomal dominant inherited disease characterised by increased low-density lipoprotein cholesterol (LDL-C) levels. The functionality of four novel variants within the LDLR $5^{\prime}$ UTR and promoter located at c.-13A $>$ G, c.-101T $>C$, c. $-121 \mathrm{~T}>\mathrm{C}$ and $\mathrm{C} .-215 \mathrm{~A}>\mathrm{G}$ was investigated using in silico and in vitro assays, and a systemic bioinformatics analysis of all 36 reported promoter variants are presented. Bioinformatic tools predicted that all four variants occurred in sites likely to bind transcription factors and that binding was altered by the variant allele. Luciferase assay was performed for all the variants. Compared with wild type, the C.-101T $>C$ and c.-121T $>C$ variants showed significantly lower mean ( \pm SD) luciferase activity $(64 \pm 8$ and $72 \pm 8 \%$, all $P<0.001)$, suggesting that these variants are causal of the FH phenotype. No significant effect on gene expression was seen for the c.-13A $>G$ or c.-215A $>G$ variants $(96 \pm 15$ and 100 $\pm 12 \%$ ), suggesting these variants are not FH causing. Similar results were seen for the $c .-101 T>C$ and $c .-121 T>C$ variants in lipid-depleted serum. However, a significant reduction in luciferase activity was seen in the c.-215A $>$ G variant in lipid-depleted serum. Electrophoretic-mobility shift assays identified allele-specific binding of liver (hepatoma) nuclear proteins to $\mathrm{C} .-121 \mathrm{~T}>\mathrm{C}$ and suggestive differential binding to c.-101T $>C$ but no binding to $\mathrm{c} .-215 \mathrm{~A}>\mathrm{G}$. These data highlight the importance of in vitro testing of reported $L D L R$ promoter variants to establish their role in $\mathrm{FH}$. The functional assays performed suggest that the c. $-101 T>C$ and c.-121T $>C$ variants are pathogenic, whereas c.-13A $>G$ variant is benign, and the status of $c .-215 A>G$ remains unclear.

European Journal of Human Genetics (2015) 23, 790-795; doi:10.1038/ejhg.2014.199; published online 24 September 2014

\section{INTRODUCTION}

Familial hypercholesterolaemia (FH) is an autosomal dominant disease characterised by high levels of low-density lipoprotein cholesterol (LDL-C) ${ }^{1}$ that leads to an increased risk of premature coronary heart disease. ${ }^{1}$ Recent guidelines for the identification and management of patients with $\mathrm{FH}$ recommend that mutation information should be used to test all first-degree relatives to find carriers and offer them lifestyle and therapeutic interventions, such as lipid-lowering statin treatment, to reduce their risk of early heart disease. ${ }^{2}$ To date, variants in three genes have been implicated in $\mathrm{FH}$, low-density lipoprotein receptor $(L D L R)$, apolipoprotein $-B(A P O B)$ and proprotein convertase subtilisin/kexin 9 (PCSK9). ${ }^{3,4} L D L R$ variants are the most common cause of $\mathrm{FH}$, with $>1200$ variants identified throughout the gene (UCL LDLR variant database: https://grenada.lumc.nl/LOVD2/ UCL-Heart/home.php?select_db $=$ LDLR). ${ }^{5,6}$ At least 36 variants have been described in the 5 'untranslated region (UTR) and promoter of the $L D L R$ gene (Table 1), many of which have been shown to alter the binding of key transcription factors involved in the control of $L D L R$ expression, such as Sterol regulatory element-binding protein (SREBP), specificity protein 1 (Sp1) and cAMP responsive elementbinding protein $(\mathrm{CREB}))^{7-9}$ As not all identified variants will be causing FH, it is important that reported variants are tested for their pathogenicity. The functionality of four variants identified in the literature $^{6}$ in patients with a diagnosis of heterozygous $\mathrm{FH}$ within the
LDLR 5'UTR (c.-13A > G) or promoter (c.-101T > C, c.-121T >C and c.-215A $>$ G) were analysed using in silico and in vitro assays. Previous in vitro luciferase results have shown that the variant c. $-120 \mathrm{C}>\mathrm{T}$ had a significant reduction in luciferase activity compared with the wild type. ${ }^{10}$ This variant was used as a positive control for these experiments. The location of known transcription factor binding sites in the $L D L R$ promoter and the positions of the tested variants are shown in Figure 1.

\section{MATERIALS AND METHODS}

Subjects

The c.-101T $>$ C and c.-215A $>$ G variants were identified by the NE Thames Regional Genetics Service laboratories, Great Ormond Street Hospital (GOSH), in patients with a clinical diagnosis of heterozygous $\mathrm{FH}$ according to the UK criteria. ${ }^{1}$ The c.- $13 \mathrm{~A}>\mathrm{G}$ and c.- $121 \mathrm{~T}>\mathrm{C}$ variants were reported in FH patients in the literature. ${ }^{11,12}$ Standard nomenclature was used for describing variants (www.hgvs.org), and the locus reference genomic sequence LRG_274 for LDLR was used.

\section{In silico analysis}

Two transcription factor prediction tools were used: MatInspector (www.genomatrix.de) and MATCH (http://www.gene-regulation.com/pub/programs.html\#match). ${ }^{13,14}$ These tools were used to identify potential transcription factor binding sites in the region where the variants in the $L D L R$ promoter were found. The matrix score calculates a match between the sequence and the matrix. The scores range from 0 to 1 , with 1 indicating an exact match.

${ }^{1}$ Centre for Cardiovascular Genetics, British Heart Foundation Laboratories, Institute Cardiovascular Science, University College London Medicine School, London, UK; ${ }^{2}$ NE Thames Regional Genetics Service, Great Ormond Street Hospital, London, UK

${ }^{*}$ Correspondence: Professor SE Humphries, Centre for Cardiovascular Genetics, British Heart Foundation Laboratories, Institute Cardiovascular Science, University College London Medicine School, The Rayne Building, 5 University Street, London WC1E 6JF, UK. Tel: +44 (0)20 7679 6962; Fax: +44 (0)20 7679 6212; E-mail:rmhaseh@ucl.ac.uk Received 28 November 2013; revised 26 June 2014; accepted 9 July 2014; published online 24 September 2014 
Table 1 Location of all reported variants $270 \mathrm{bp}$ upstream from LDLR start of translation, with reported effect on promoter activity and homology across human and 6 primate and 6 mammalian species (variants tested in this study are shown in bold)

\begin{tabular}{|c|c|c|c|c|}
\hline Variant & Promoter activity (\% of wt) & Location & Sequence homology ${ }^{a}$ & Reference \\
\hline c. $-268 G>T$ & In $\mathrm{FH}$ and normal & $1 \mathrm{bp}$ from FP1 & $7 / 8$ & 20 \\
\hline c. $-220 \_-221$ ins A & Not tested & $\mathrm{FP} 1$ & NA & 21 \\
\hline c. $-217 \mathrm{C}>\mathrm{T}$ & $160 \%$ Luciferase & $2 \mathrm{bp}$ from FP1 & $13 / 13$ & 20 \\
\hline c. $-215 A>G$ & $100 \%$ Luciferase & $4 \mathrm{bp}$ from FP1 & $11 / 12$ & 6 \\
\hline c. $-208 \mathrm{~A}>\mathrm{T}$ & $100 \%$ Luciferase & Between SREBP1 and FP1 & $12 / 12$ & 15 \\
\hline c. $-206 C>T$ & Not tested & Between SREBP1 and FP1 & $13 / 13$ & 22 \\
\hline c.-193_-186delinsTG & Not tested & SREBP1 & NA & 23 \\
\hline c. $-188 \mathrm{C}>\mathrm{T}$ & Not tested & SREBP1 & $13 / 13$ & 23 \\
\hline c.-185_-183delCTT & $10 \%$ Luciferase & SREBP1 & NA & 24 \\
\hline c. $-156 \mathrm{C}>\mathrm{T}$ & Not tested ${ }^{\mathrm{b}}$ & SREBP2 & $13 / 13$ & 25 \\
\hline c.-155_-154delinsTTCTGCAAACTCCT & $11 \%$ Luciferase & SREBP2 & NA & 15 \\
\hline c. $-153 C>T$ & Not tested & SREBP2 & $13 / 13$ & 26 \\
\hline c. $-152 \mathrm{C}>\mathrm{T}$ & $40 \%$ Luciferase & SREBP2 & $13 / 13$ & 20 \\
\hline c. $-146 C>A$ & Not tested & Between SREBP2 and SP1 & $13 / 13$ & 27 \\
\hline c. $-142 \mathrm{C}>\mathrm{T}$ & $20 \%$ Luciferase & SP1 & $13 / 13$ & 28 \\
\hline c. $-140 \mathrm{C}>\mathrm{G}$ & 7\% Luciferase & SP1 & $13 / 13$ & 15 \\
\hline c. $-140 \mathrm{C}>\mathrm{T}$ & $6 \%$ Luciferase & SP1 & $13 / 13$ & 15 \\
\hline c. $-139 \mathrm{C}>\mathrm{A}$ & Not tested & SP1 & $13 / 13$ & 23 \\
\hline c. $-139 \mathrm{C}>\mathrm{G}$ & $26 \%$ Luciferase & SP1 & $13 / 13$ & 8 \\
\hline c.-138delT & 24\% LDLR activity & SP1 & $12 / 13$ & 29 \\
\hline c. $-138 \mathrm{~T}>\mathrm{C}$ & $25 \%$ Luciferase & SP1 & $12 / 13$ & 30 \\
\hline c. $-137 \mathrm{C}>\mathrm{T}$ & Not tested ${ }^{c}$ & SP1 & $13 / 13$ & 31 \\
\hline c. $-136 \mathrm{C}>\mathrm{G}$ & $12 \%$ Luciferase & SP1 & $13 / 13$ & 15 \\
\hline c. $-136 \mathrm{C}>\mathrm{T}$ & $5 \%$ CAT assay & SP1 & $13 / 13$ & 32 \\
\hline c. $-135 \mathrm{C}>\mathrm{G}$ & Not tested ${ }^{\mathrm{d}}$ & $\mathrm{SP} 1$ & $13 / 13$ & 31 \\
\hline c. $-121 \mathrm{~T}>\mathrm{C}$ & $72 \%$ Luciferase & Between TATA box and SP1 & $13 / 13$ & 12 \\
\hline c. $-120 \mathrm{C}>\mathrm{T}$ & $3 \%$ Luciferase & Between TATA box and SP1 & $13 / 13$ & 26 \\
\hline C. $-101 \mathrm{~T}>\mathrm{C}$ & $64 \%$ Luciferase & TATA BOX & $12 / 12$ & 6 \\
\hline c. $-88 G>A$ & $100 \%$ Luciferase & 5'UTR & $12 / 12$ & 15 \\
\hline c. $-68 \mathrm{~A}>\mathrm{C}$ & Not tested & 5'UTR & $5 / 11$ & 33 \\
\hline c. $-36 \mathrm{~T}>\mathrm{G}$ & $100 \%$ Luciferase & 5'UTR & $6 / 10$ & 15 \\
\hline c. $-23 A>C$ & Not tested & 5'UTR & $7 / 12$ & 34 \\
\hline c.-22delC & Not tested & 5'UTR & $10 / 12$ & 35 \\
\hline c. $-14 C>A$ & Not tested & 5'UTR & $9 / 12$ & 27 \\
\hline c. $-13 A>G$ & $100 \%$ Luciferase & 5'UTR & $8 / 13$ & 11 \\
\hline c. $-5 C>T$ & Not tested & 5'UTR & $7 / 11$ & 23 \\
\hline
\end{tabular}

Abbreviations: FP1, Foot Print 1; SREBP1, sterol regulating element-binding protein 1; SP1, Specificty Protein 1.

Where the base for the sequence was not present in a particular species, the species was discounted from the total.

The NG_009060.1 reference sequence was used.

a LDLR promoter sequence conservation between Human, Pan troglodytes, Gorilla gorilla, Pongo abelii, Macaca mulatta, Callithrix jacchus, Oryctolagus cuniculus, Mus musculus, Rattus norvegicus, Bos taurus, Sus scrofa, Canis lupus familiaris and Equus caballus (http://www.ensembl.org/Homo_sapiens/Gene/Compara_Alignments?align $=609 \& d b=c o r e \& g=E N S G 00000130164 \& r=19 \%$ 3A11200057-11244506\&t = ENST00000252444).

${ }^{\mathrm{b}}$ Cosegregation with $\mathrm{FH}$ reported.

c5-15\% LDLR activity (when compound heterozygote with c.1222G > A, p.(Glu408Lys)).

${ }^{d} 5-15 \%$ LDLR activity in homozygote.

\section{Cloning of $L D L R$ promoter}

The c.- $600 \mathrm{bp}$ to c.-5 bp region of the $L D L R$ promoter was cloned into the pGL2-basic vector using the HindIII restriction site. ${ }^{8}$ This was used as a template for site-directed mutagenesis using the QuikChange Lightning SiteDirected Mutagenesis Kit (210518-5; Agilent Stratagene Technologies, Stockport, UK). Site-directed mutagenesis was completed to create plasmids containing the c.-13G, c.-101C, c.-121C and c.-215G alleles. All constructs were confirmed by sequencing. The plasmids were transfected into Huh7 cells, a human hepatoma liver cell line (01042712; European collection of cell cultures (ECACC)). The cells were grown using Dulbecco's modified Eagle's medium (DMEM) with 10\% FBS, or 10\% lipid-depleted serum (from GENTAUR Europe BVBA, Aachen, Germany; FB-1090LF/100).

\section{Transfection and luciferase assay}

Transfection was prepared when a confluence of $80 \%$ was reached. The pUC18 and pGL3 control were used as negative and positive controls, respectively. The cells were transfected using Lipofectamine 2000 (Invitrogen, Paisley, UK) and Opti-MEM serum-free medium according to the manufacturer's instructions and as previously described. ${ }^{7}$ Cells were lysed using Passive Lysis Buffer (Promega, Southampton, UK) and luciferase expression was determined using the Dual Luciferase Reporter Assay System (Promega), and measured in the Tropix TR717 Microplate Luminometer (PE Applied Biosystems, Paisley, UK). Luciferase activity was determined as the mean of 8-12 transfections with the assay performed in triplicate. EMSAs were carried out, as previously described, to determine transcription factor binding within the variant site. ${ }^{8}$

\section{RESULTS}

In silico analysis indicated that all of the variants were likely to be within transcription factor binding sites (Supplementary Table 1). With the exception of the predicted CREB site at c.-101T $>$ C, none of these have been reported to be involved in control of transcription of 


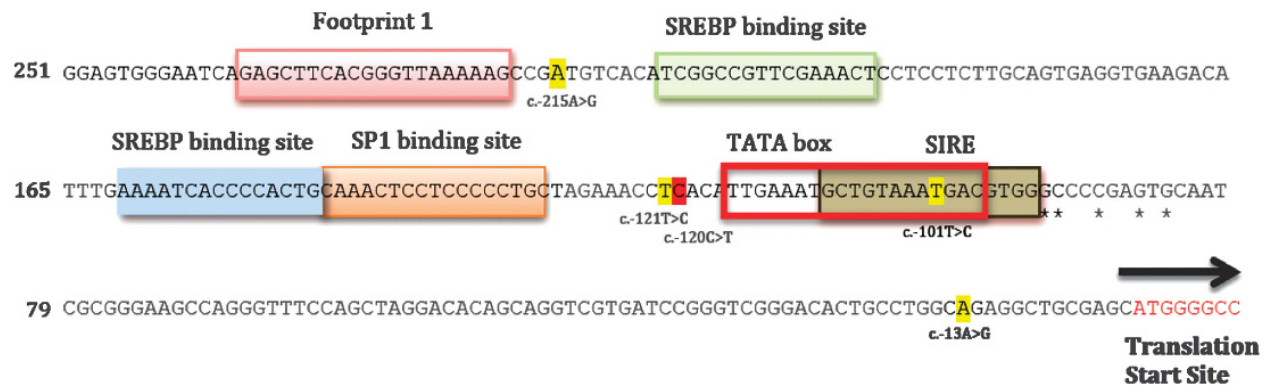

Figure 1 Illustration of the promoter and 5'UTR region of the LDLR gene. Highlighted boxes illustrate known binding sites. Variants examined in this study are highlighted in red, and highlighted in yellow is the positive control used. The start sites for transcription are indicated by *. The numbers to the left indicate the nucleotide number.

the LDLR gene. The minor differences in predicted scores for the variants at the c.-121 site are unlikely to be of biological significance. For three of the variants (c.-13A $>$ G, c. $-101 T>C$ and c. $-215 A>G$ ), one or both of the programmes used predicted that binding was likely to be significantly modified by the presence of the variant allele, for example, the predicted binding of CREB to c.-101T but not c.-101C, and the binding of HIFIA to c.-101C and not c.-101T. However, although the c.-13G allele showed the abolition of two transcription factors, this binding was not verified in MATCH. Moreover, in MatInspector, the c.-120C $>\mathrm{T}$ variant showed an abolition of OCT1 binding site, indicating a biological significance. However, this result was not seen in the MATCH results.

Luciferase assays were performed to investigate the $L D L R$ expression in the promoter sequences containing the variant alleles compared with the wild type. These assays were repeated in multiples of 8-12 for each variant and are presented as the average of three independent runs. Compared with the wild type, the c.-13G and c.-215G alleles showed a (mean \pm SD) $96 \pm 15$ and $100 \% \pm 12 \%$ level of luciferase activity, indicating that this variant is unlikely to be $\mathrm{FH}$ causing. The c. $-101 \mathrm{~T}>\mathrm{C}$ and c. $-121 \mathrm{~T}>\mathrm{C}$ variants showed a $64 \pm 8$ and $72 \pm 8 \%$ lower luciferase activity compared with the wild type (all $P<0.001$; Figure $2 \mathrm{a}$ ), indicating that all these variants are likely to be $\mathrm{FH}$ causing. The c. $-120 \mathrm{C}>\mathrm{T}$ variant was then used as a positive control, showing $83 \%$ expression as compared with the wild type $(P=0.001)$. All transfection assays were repeated using cells grown in 10\% lipiddepleted serum in order to induce $L D L R$ promoter expression. ${ }^{8}$ This resulted in a 2.5-fold higher expression of the wild-type construct, with similar increases seen for all variants (data not shown). However, as shown in Figure 2b, compared with the wild-type construct, the pattern of expression seen was similar, with the c.-13G allele showing a $96 \pm 22 \%$ level of luciferase activity, indicating that this variant is unlikely to be FH causing, whereas c.-101T $>\mathrm{C}$ and c. $-121 \mathrm{~T}>\mathrm{C}$ variants showed $48 \pm 8$ and $49 \pm 6 \%$, respectively (all $P<0.001$ ). Remarkably, the c.-215G allele showed a similar and significant reduction in luciferase activity in the absence of lipid $(48 \pm 10 \%$; $P<0.001$ )

EMSAs were used in an attempt to identify nuclear factors binding differentially to the c.-101T $>$ C, c. $-121 \mathrm{~T}>\mathrm{C}$ and c. $-215 \mathrm{~A}>\mathrm{G}$ variants. Extracts from a hepatocarcinoma cell line Huh7 were used, with a probe for the SREBP as a positive control. As shown in Supplementary Figure 1, the positive control provided a strong band shift as well as several nonspecific bands. Neither alleles of the c. $-215 \mathrm{~A}>\mathrm{G}$ probe showed a specific additional band; however, the c.-121T allele showed an additional band shift compared with the c.-121C allele. For the c.-101 probes, both showed evidence of a specific high-molecular-weight band, with the c.-101C allele showing an additional faint band (indicated by an arrow in Supplementary Figure 1).

\section{DISCUSSION}

In order to correctly report to a referring clinician whether or not an identified promoter variant is likely to be $\mathrm{FH}$ causing, bioinformatics predictions may be helpful, and although evidence that the variant affects binding of a nuclear protein by an EMSA may be suggestive, an in-vitro assay of the effect on transcriptional strength provides the strongest evidence. Four reported variants identified in FH patients within the LDLR 5'UTR and promoter were investigated. ${ }^{6}$ The c.- $13 \mathrm{~A}>\mathrm{G}$ variant showed no difference in luciferase activity, suggesting it is not $\mathrm{FH}$ causing, whereas c.-101C and c.-121C showed a significant lower expression, suggesting that these variants are likely to be pathogenic and causal of the $\mathrm{FH}$ phenotype seen in these patients, and the status of c. $-215 \mathrm{~A}>\mathrm{G}$ remains unclear.

The bioinformatic programmes were not helpful in this instance. Both programmes (MatInspector and $\mathrm{MATCH}$ ) suggested that the c. $-13 \mathrm{~A}>\mathrm{G}$ variant destroys binding for a nuclear transcription factor and yet this variant did not affect luciferase activity. This is in agreement with previous studies that have shown no causal effect of variants within the $5^{\prime} \mathrm{UTR}$ region. ${ }^{15}$ Both programmes used also suggested that transcription factors would bind to the DNA sequences and that the variants would destroy binding. This is particularly clear for the c.-101T $>\mathrm{C}$ allele that is located in the TATA-box and a previously documented CREB binding site. ${ }^{7}$ Moreover, there were major differences between the two programmes; for example, although MatInspector showed a high score for the binding of two transcription factors, MATCH did not find any binding sites. Of the identified transcription factor binding sites (Supplementary Table 1), CREB is involved in $L D L R$ expression. The SIRE (sterol-independent regulatory element), located c.-94 to c.-110 of the promoter, binds CREB in a cholesterol-independent manner. ${ }^{7}$ CREB was predicted to bind to the wild-type sequence, but was abolished in the variant sequence. Moreover, another predicted transcription factor, OCT1, has been implicated as being involved in lipoprotein lipase $(L P L)$ expression, and is critical for hydrolysis of triglycerides in lipoproteins. ${ }^{16,17}$ Although OCT1 was predicted to bind by MatInspector and MATCH, there are no major differences in the scores for the variant and wild-type binding sites. These algorithms lack the complexity to take account of physiological conditions. The binding of transcription factors to a specific DNA sequence is dependent on various intracellular and extracellular stimuli within a cell. Therefore, although some transcription factors are predicted to bind to these sequences (with scores as high as 1.0), this does not take into account in vivo conditions such as chromatin structure, and are hence not ideal representations of what 
a

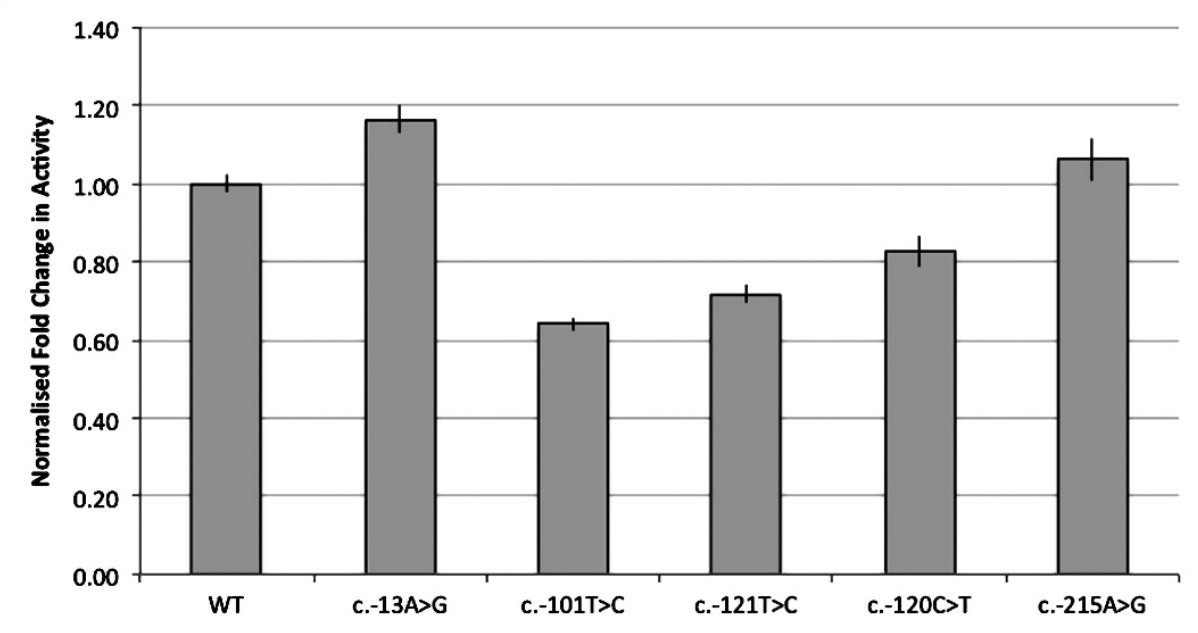

\begin{tabular}{|c|c|c|c|c|c|c|}
\hline & WT & C.-13A>G & C.-101T>C & C.-121T>C & C.-120C>T & C.-215A>G \\
\hline average & 1.00 & 1.17 & 0.64 & 0.72 & 0.83 & 1.06 \\
\hline SD & 0.23 & 0.28 & 0.17 & 0.22 & 0.20 & 0.47 \\
\hline SEM & 0.02 & 0.04 & 0.02 & 0.02 & 0.04 & 0.05 \\
\hline p values vs WT & & 0.00011 & $2.810^{-24}$ & $1.0010^{-15}$ & 0.0011 & 0.25 \\
\hline p value -121/-120 & & & & & 0.026 & \\
\hline number of repeats & 100 & 56 & 85 & 94 & 24 & 76 \\
\hline
\end{tabular}

b

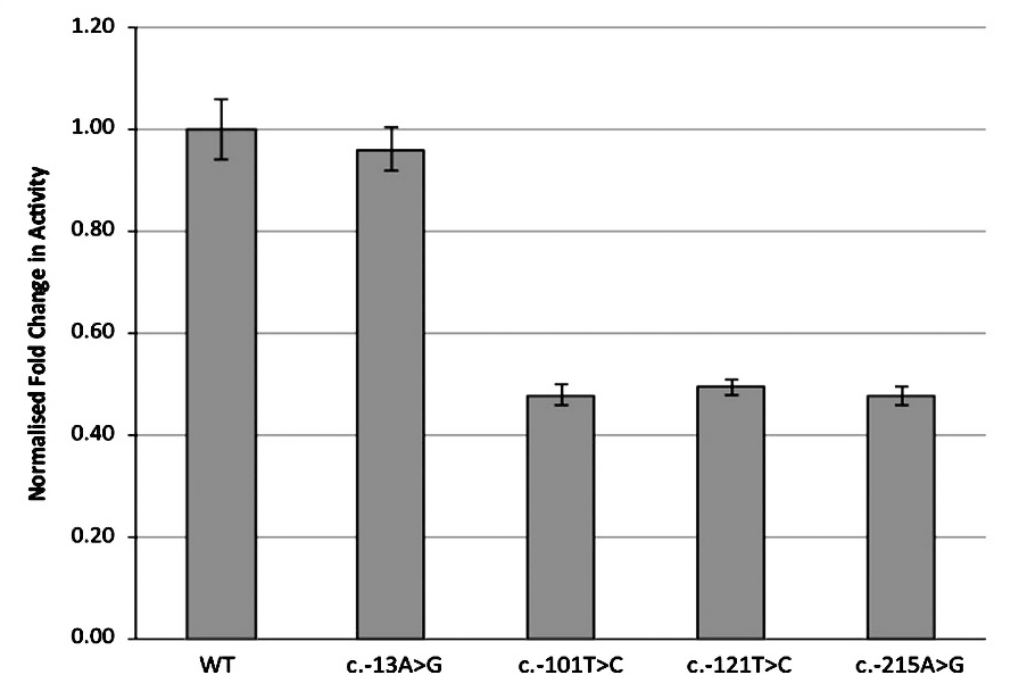

Figure 2 (a) Measurement of promoter activity using luciferase assay. Fragments containing the wild type and mutant were cloned into the pGL2 promoter, transfected into Huh7 cells and cultured using DMEM medium. Data were obtained in medium supplemented with $10 \%$ bovine calf serum. The mutants were compared with the wild type, normalised to the value of 1 . These results are the average of 8-12 repeats in 3 independent experiments. Error bars indicate SEM. (b) Measurement of promoter activity using luciferase assay. Data were obtained in medium supplemented with $10 \%$ lipid-depleted bovine calf serum.

occurs in the cell. Bioinformatics tools should not be used in isolation but as a guide for experimental research.

The EMSA assay was also inconclusive: one out of the three functional sites determined by luciferase assay showed no evidence of binding of a nuclear protein, and this may be because of the protein requiring a longer binding site than the probe provided or nonphysiological binding conditions. Although we are not aware of any direct evidence for a role of HIF1A in the control of $L D L R$ expression, this factor is involved in expression of PCSK $9,{ }^{18}$ a key gene involved in cholesterol metabolism, and hence the prediction of its binding here may be correct.
In order to put the present findings in context, we have carried out a systematic review of the published promoter and $5^{\prime} \mathrm{UTR}$ variants and examined the functional assay data reported. Including the four variants examined here, there are 36 variants reported, of which 20 have been directly tested using reporter assays, usually luciferase (Table 1). Of the four variants identified after the upstream SREBP1 binding site (which extends from c.-193 to c.-107), two have been tested and one (c.-217C $>\mathrm{T}$ ) reported to have higher transcriptional activity and one (c.-208A $>$ T) with no effect on luciferase activity, and thus neither are FH causing. The variant tested here at c.-215A $>$ G has a modest effect on transcription and no EMSA binding was detected. 
This region contains sequences involved in the Footprint 1 element responsible for enhancement of $L D L R$ transcription, with the key nucleotides identified at c. -218 , c. -219 and c. $-231,{ }^{19}$ but no variants at these sites have been reported so far. Of the 23 variants between c.-207 and c.-90, where the binding sites for the key transcription factors SREBP1, SP1 and the TATA box CREBP are located, 14 have been tested and all found to have a profound effect on luciferase levels. Excluding the two examined here, the percentage of wild-type activity reported ranges from 0 to $40 \%$ (mean 15\%). The modestly lower $\sim 50 \%$ luciferase noted for the variants tested here (c.-215A $>\mathrm{G}$, c. $-121 \mathrm{~T}>\mathrm{C}$ and c. $-101 \mathrm{~T}>\mathrm{C}$ ) therefore raises the possibility that these may not be FH causing, although the data obtained are based on 8-12 replicates and three repeat experiments, and the lower transcriptional data are statistically robust. To address this, we have used c.-120C $>$ T as a positive control that has been reported to have only $3 \%$ luciferase activity compared with the wild-type promoter sequence. ${ }^{10}$ In our hands, we observe that the variant has $83 \pm 9 \%$ activity compared with the wild type, confirming that the variant is $\mathrm{FH}$ causing but that the effect is not so drastic as previously observed. This could be because of the differences in cell culture conditions; for example, Francova et al ${ }^{10}$ used the HepG2 cell line compared with the Huh7 hepatoma cells that we used. However, the reduction in luciferase activity observed with the novel variants tested here are similar to the reported FH-causing c. $-120 \mathrm{C}>\mathrm{T}$ variant, strongly supportive of a causal effect of our variants. Finally, of the eight variants located after the start of transcription (c.-93 to c.-79), two have been tested and, as with the c. $-13 \mathrm{~A}>\mathrm{G}$ tested here, none have a significant effect on promoter strength. ${ }^{15}$ Our data suggest that although there is no effect on luciferase activity for the c.-215G allele, there was a significant reduction in luciferase activity when supplemented in lipid-depleted conditions. In lipid-depleted conditions, SREBPs activate the transcription of $L D L R$ by binding to SRE. Therefore, when lipid levels are low, $L D L R$ expression is increased. However for the c.-215G allele construct, in lipid-depleted conditions, luciferase expression was reduced compared with the wild-type construct. Therefore, because lipid-depleted conditions mimic what occurs in vivo, these data support the view that this variant is possibly causal of the $\mathrm{FH}$ phenotype, but the status of this variant is still unclear, and co-segregation analysis in the relatives of this patient may resolve this issue. The data obtained from this study have been submitted to the UCL LDLR variant database (https://grenada.lumc.nl/LOVD2/UCLHeart/home.php?select_db=LDLR).

There is high cross-species conservation across the whole of the $L D L R$ promoter region and all the four sites tested here show strong conservation across six primate and six mammalian species (Table 1). It is noteworthy that the lowest sequence conservation is seen in sites in the $5^{\prime} \mathrm{UTR}$, where the majority of the tested variants appear not to affect promoter strength, suggesting that this region does not have a critical role in control of gene expression. A limitation of the data reported here is that we do not have any relative samples available from these probands as the variants were identified from published reports, ${ }^{11,12}$ or from single samples sent to the diagnostic laboratory. Co-segregation of the variant with elevated lipid levels in relatives would strengthen the inference that they are FH causing. For all patients, DNA was tested for the common $\mathrm{FH}$-causing mutations in the $A P O B$ and PCSK9 genes and for the Portuguese patient the entire $P C S K 9$ gene was screened, but it remains a formal possibility that their elevated LDL-C levels may be caused by an unidentified variant in these genes. Finally, for the EMSAs we did not carry out detailed competition or supershift experiments to confirm the specificity or identity of the nuclear proteins binding to the alleles, as in our experience these type of experiments are difficult to quantify with accuracy and often give equivocal results that may not be of direct relevance to the in vivo situation because the examined element is not embedded within the full chromatin context.

In vitro luciferase reporter assays for identified promoter variants are important in determining the pathogenicity of promoter variants, allowing diagnostic reporting with greater confidence and subsequent cascade testing in the relatives of patients with these variations. This study highlights the difficulty of relying solely on in silico methods for the prediction of the pathogenicity of DNA sequence variants. Ideally, functional assays should be used to determine effects; however, the dilemma faced by many diagnostic service laboratories is that such tests are expensive and time consuming, and the relevant expertise and resources are rarely available in-house. Therefore, close links with research groups are key to the transfer of expert knowledge to diagnostic service laboratories. In conclusion, the functional assays performed indicate that c.-101T $>\mathrm{C}$ and c.-121T $>\mathrm{C}$ are likely to be pathogenic, whereas the c.-13A $>\mathrm{G}$ variant appears to be benign and the status of c. $-215 \mathrm{~A}>\mathrm{G}$ remains unclear.

\section{CONFLICT OF INTEREST}

The authors declare no conflict of interest.

\section{ACKNOWLEDGEMENTS}

SEH holds a Chair funded by the British Heart Foundation. SEH, SL and JP were supported by the BHF (PG08/008). This work was supported by the British Heart Foundation.

1 Marks D, Thorogood M, Neil HA, Humphries SE: A review on the diagnosis, natural history, and treatment of familial hypercholesterolaemia. Atherosclerosis 2003; 168 $1-14$

2 Nordestgaard BG, Chapman MJ, Humphries SE et al: Familial hypercholesterolaemia is underdiagnosed and undertreated in the general population: guidance for clinicians to prevent coronary heart disease: consensus statement of the European Atherosclerosis Society. Eur Heart J 2013; 34: 3478-90a.

3 Soutar AK, Naoumova RP: Mechanisms of disease: genetic causes of familia hypercholesterolemia. Nature clinical practice. Cardiovasc Med 2007; 4: 214-225.

4 Varret M, Abifadel M, Rabes JP, Boileau C: Genetic heterogeneity of autosomal dominant hypercholesterolemia. Clin Genet 2008; 73: 1-13.

5 Leigh SE, Foster AH, Whittall RA, Hubbart CS, Humphries SE: Update and analysis of the University College London low density lipoprotein receptor familial hypercholesterolemia database. Ann Hum Genet 2008; 72 (Pt 4): 485-498.

6 Usifo E, Leigh SE, Whittall RA et al: Low-density lipoprotein receptor gene familial hypercholesterolemia variant database: update and pathological assessment. Ann Hum Genet 2012; 76: 387-401

7 Liu J, Ahlborn TE, Briggs MR, Kraemer FB: Identification of a novel sterol-independent regulatory element in the human low density lipoprotein receptor promoter. $\mathrm{J}$ Biol Chem 2000; 275: 5214-5221.

8 Smith AJ, Ahmed F, Nair D et al: A functional mutation in the LDLR promoter $(-139 \mathrm{C}>\mathrm{G})$ in a patient with familial hypercholesterolemia. Eur J Hum Genet 2007; 15: $1186-1189$.

9 Natesampillai S, Fernandez-Zapico ME, Urrutia R, Veldhuis JD: A novel functional interaction between the Sp1-like protein KLF13 and SREBP-Sp1 activation complex underlies regulation of low density lipoprotein receptor promoter function. J Biol Chem 2006; 281: 3040-3047.

10 Francova H, Trbusek M, Zapletalova P, Kuhrova V: New promoter mutations in the low-density lipoprotein receptor gene which induce familial hypercholesterolaemia phenotype: molecular and functional analysis. J Inherit Metab Dis 2004; 27: 523-528.

11 Medeiros AM, Alves AC, Francisco V, Bourbon M: Update of the Portuguese Familial Hypercholesterolaemia Study. Atherosclerosis 2010; 212: 553-558.

12 Taylor A, Wang D, Patel $\mathrm{K}$ et al: Mutation detection rate and spectrum in familia hypercholesterolaemia patients in the UK pilot cascade project. Clin Genet 2010; 77 572-580.

13 Cartharius K, Frech K, Grote K et al: MatInspector and beyond: promoter analysis based on transcription factor binding sites. Bioinformatics 2005; 21: 2933-2942.

$14 \mathrm{Kel}$ AE, Gossling E, Reuter I, Cheremushkin E, Kel-Margoulis OV, Wingender E: MATCH: a tool for searching transcription factor binding sites in DNA sequences. Nucleic Acids Res 2003; 31: 3576-3579. 
15 De Castro-Oros I, Pampin S, Bolado-Carrancio A et al: Functional analysis of LDLR promoter and $5^{\prime}$ UTR mutations in subjects with clinical diagnosis of familial hypercholesterolemia. Hum Mutat 2011; 32: 868-872.

16 Yang WS, Nevin DN, Peng R, Brunzell JD, Deeb SS: A mutation in the promoter of the lipoprotein lipase (LPL) gene in a patient with familial combined hyperlipidemia and low LPL activity. Proc Natl Acad Sci USA 1995; 92: 4462-4466.

17 Nakshatri H, Nakshatri P, Currie RA: Interaction of Oct-1 with TFIIB. Implications for a novel response elicited through the proximal octamer site of the lipoprotein lipase promoter. J Biol Chem 1995; 270: 19613-19623.

18 Li H, Dong B, Park SW, Lee HS, Chen W, Liu J: Hepatocyte nuclear factor 1alpha plays a critical role in PCSK9 gene transcription and regulation by the natural hypocholesterolemic compound berberine. J Biol Chem 2009; 284: 28885-28895.

19 Dhawan P, Chang R, Mehta KD: Identification of essential nucleotides of the FP1 element responsible for enhancement of low density lipoprotein receptor gene transcription. Nucleic Acids Res 1997; 25: 4132-4138.

20 Scholtz CL, Peeters AV, Hoogendijk CF et al: Mutation $-59 c->t$ in repeat 2 of the LDL receptor promoter: reduction in transcriptional activity and possible allelic interaction in a South African family with familial hypercholesterolaemia. Hum Mol Genet 1999; 8: 2025-2030.

21 Bodamer OA, Bercovich D, Schlabach M, Ballantyne C, Zoch D, Beaudet AL: Use of denaturing HPLC to provide efficient detection of mutations causing familial hypercholesterolemia. Clin Chem 2002; 48: 1913-1918.

22 Lind S, Rystedt E, Eriksson M, Wiklund O, Angelin B, Eggertsen G: Genetic characterization of Swedish patients with familial hypercholesterolemia: a heterogeneous pattern of mutations in the LDL receptor gene. Atherosclerosis 2002; 163: 399-407.

23 Fouchier SW, Kastelein JJ, Defesche JC: Update of the molecular basis of familial hypercholesterolemia in The Netherlands. Hum Mutat 2005; 26: 550-556.

24 Peeters AV, Kotze MJ, Scholtz CL et al: A 3-basepair deletion in repeat 1 of the LDL receptor promoter reduces transcriptional activity in a South African Pedi. J Lipid Res 1998; 39: 1021-1024.

25 Dedoussis GV, Genschel J, Bochow B et al: Molecular characterization of familial hypercholesterolemia in German and Greek patients. Hum Mutat 2004; 23: 285-286.

26 Francova H, Trbusek M, Zapletalova P, Kuhrova V: New promoter mutations in the lowdensity lipoprotein receptor gene which induce familial hypercholesterolaemia phenotype: molecular and functional analysis. J Inherit Metab Dis 2004; 27: 523-528.

27 Day IN, Whittall RA, O'Dell SD et al: Spectrum of LDL receptor gene mutations in heterozygous familial hypercholesterolemia. Hum Mutat 1997; 10: 116-127.
28 Mozas P, Galetto R, Albajar M, Ros E, Pocovi M, Rodriguez-Rey JC: A mutation $(-49 \mathrm{C}>\mathrm{T})$ in the promoter of the low density lipoprotein receptor gene associated with familial hypercholesterolemia. J Lipid Res 2002; 43: 13-18.

29 Dedoussis GV, Pitsavos C, Kelberman D et al: FH-Pyrgos: a novel mutation in the promoter (-45delT) of the low-density lipoprotein receptor gene associated with familial hypercholesterolemia. Clin Genet 2003; 64: 414-419.

30 Sun XM, Neuwirth C, Wade DP, Knight BL, Soutar AK: A mutation (T-45C) in the promoter region of the low-density-lipoprotein (LDL)-receptor gene is associated with a mild clinical phenotype in a patient with heterozygous familial hypercholesterolaemia (FH). Hum Mol Genet 1995; 4: 2125-2129.

31 Hobbs HH, Brown MS, Goldstein JL: Molecular genetics of the LDL receptor gene in familial hypercholesterolemia. Hum Mutat 1992; 1: 445-466.

32 Koivisto UM, Palvimo JJ, Janne OA, Kontula K: A single-base substitution in the proximal Sp1 site of the human low density lipoprotein receptor promoter as a cause of heterozygous familial hypercholesterolemia. Proc Natl Acad Sci USA 1994; 91: 10526-10530.

33 Laurie AD, Scott RS, George PM: Genetic screening of patients with familia hypercholesterolaemia (FH): a New Zealand perspective. Atheroscler Supp/ 2004; 5: 13-15.

34 Mozas P, Castillo S, Tejedor D et al: Molecular characterization of familial hypercholesterolemia in Spain: identification of 39 novel and 77 recurrent mutations in LDLR. Hum Mutat 2004; 24: 187.

35 Sozen MM, Whittall R, Oner $\mathrm{C}$ et al: The molecular basis of familial hypercholesterolaemia in Turkish patients. Atherosclerosis 2005; 180: 63-71.

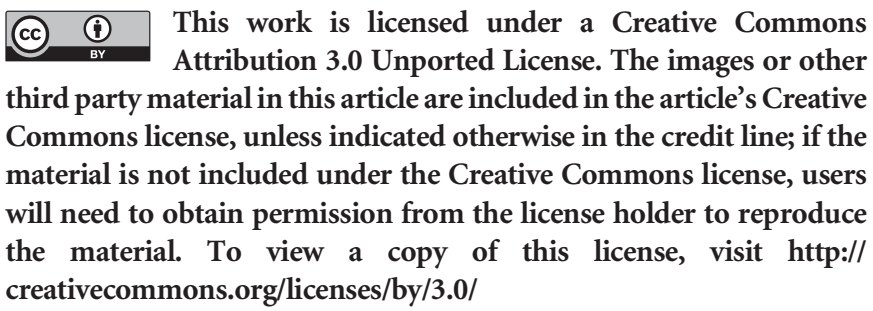

Supplementary Information accompanies this paper on European Journal of Human Genetics website (http://www.nature.com/ejhg) 